\title{
Lymphatic and blood microvasculature organisation in pulmonary sarcoid
} granulomas

\author{
M. Kambouchner*, D. Pirici”, J-F. Uhl`, L. Mogoanta\#, D. Valeyre ${ }^{+}$and J-F. Bernaudin ${ }^{\S}$
}

ABSTRACT: Pulmonary sarcoid granulomas are characterised by their elective distribution along collecting lymphatics. However, relationships between granulomas and intralobular lymphatics or blood microvascularisation have not been investigated. Therefore, we undertook a specific analysis of blood capillaries and lymphatics supplying sarcoid granulomas to identify additional clues to understanding the pathophysiogenesis of these lesions.

Six pulmonary samples were immunolabelled with D2-40, anti-CD34 and anti-CD31 antibodies, paying particular attention to the relationships between lymphatics and granulomas, and the pattern of blood microvessels supplying sarcoid lesions. A morphometric study of granulomas included their distance to lymphatics and a three-dimensional reconstruction of a granuloma in its lymphatic context.

Intralobular granulomas were closely associated with lymphatics; apart from a few granulomas, blood capillaries stopped at the outer border of the fibrous ring surrounding granulomas, and perigranuloma capillaries were particularly scarce.

Our observations of the lymphatic and blood microvascular environment of intralobular pulmonary sarcoid granulomas provide evidence for the critical role of lymphatics in the emergence of these lesions. Moreover, pulmonary sarcoid lesions could be considered avascular structures, thereby providing new insights into the understanding of the granuloma physiology and the distribution of blood-borne therapeutic agents.

\section{KEYWORDS: Fibrosis, lymphatics, microvessels, sarcoid granulomas}

arcoidosis is a multisystem disorder of unknown cause with thoracic involvement in up to $90 \%$ of patients [1]. Sarcoid granulomas are comprised of clusters of epithelioid and giant cells surrounded by a rim of lymphocytes and fibroblasts. A peripheral fibrous ring of varied thickness surrounds the lesions that have a tendency to coalesce, resulting in typical fibrotic nodules. The characteristic distribution of granulomas along the pulmonary collecting lymphatics, i.e. peribronchovascular spaces, interlobular septa and subpleural connective tissue, is a major diagnostic criterion [2]. Moreover, their tropism for the outer wall of pulmonary arteries or veins is well known [3].

In addition to these features, the relationships between pulmonary sarcoid granulomas and the intralobular lymphatic network or the pulmonary blood capillaries have not yet been adequately examined. Investigating these close relationships might help improve our understanding the biology of granulomas and also orient therapeutic drug research to achieve better targeted distribution. To address this issue, we systematically analysed the interface between granulomas and the lymphatic network by means of D2-40 immunohistochemical labelling or their blood microvessels with CD34 and CD31 immunolabelling [4,5]. To better discriminate their blood and lymphatic vascular supplies we specifically focused our study on selected isolated granulomas, whose location is predominantly intralobular.

\section{MATERIALS AND METHODS}

\section{Surgical lung biopsies}

Surgical lung biopsies from six patients (one female and five males; age range: 29-53 yrs) with pulmonary sarcoidosis referred to our Pulmonary Dept were retrieved from the files of the Dept of Pathology (both Hôpital Avicenne, APHP, Bobigny, France). These tissue specimens were selected because of the presence of multiple, well-defined, sarcoid granulomas scattered throughout the pulmonary parenchyma.

\section{AFFILIATIONS}

*Depts of Pathology,

+Pulmonary Medicine, Hôpital

Avicenne, AP-HP, Université Paris

13, Bobigny,

"URDIA EA 4465, Université Paris

Descartes,

${ }^{\S}$ Laboratory of Histology and Tumour Biology, Hôpital Tenon, AP-HP and ER2 Université Paris 6, Paris, France, and

\#Dept of Histology, University of Medicine and Pharmacy, Craiova, Romania.

\section{CORRESPONDENCE}

M. Kambouchner

Dept of Pathology, Hôpital Avicenne, AP-HP

Université Paris 13

93009 Bobigny Cedex

France

E-mail: marianne.kambouchner@

avc.aphp.fr

Received:

June 042010

Accepted after revision: July 052010 First published online: July 222010 
This retrospective study was conducted in accordance with French legislation concerning medical research.

\section{Tissue-section processing}

$5-\mu \mathrm{m}$ thick tissue sections were stained with haematoxylin and eosin and Masson's trichrome. For immunohistochemistry studies, sections were incubated with mouse monoclonal immunoglobulin (Ig)G antibodies to D2-40, which recognises podoplanin expressed by lymphatic vascular endothelium, anti-CD31, which recognises platelet endothelial cell adhesion molecule-1, and anti-CD34, which recognises a 115-kDa transmembrane glycoprotein $[4,5]$. The latter two are expressed on blood vascular endothelial cells [5]. 40 serial sections from one specimen were incubated only with D2-40 for the three dimensional (3D) study.

Immunolabelling was processed as previously described using an automated procedure (Ventana Medical Systems, Tucson, AZ, USA). Antigen retrieval was achieved by boiling at 95- $100^{\circ} \mathrm{C}$. Anti-D2-40 (clone 07 3611; 1/100 dilution; concentration $0.25 \mathrm{mg} \cdot \mathrm{L}^{-1}$; Dako, Trappes, France) was incubated for $32 \mathrm{~min}$ at $37^{\circ} \mathrm{C}$. Anti-CD34 (clone $963820 \mathrm{~B} ; 1 / 100$ dilution; concentration $0.5 \mathrm{mg} \cdot \mathrm{L}^{-1}$; Dako) was incubated for $60 \mathrm{~min}$ at $42^{\circ} \mathrm{C}$, and anti-CD31 (clone JC70A; $1 / 20$ dilution; concentration $515 \mathrm{mg} \cdot \mathrm{L}^{-1}$; Dako) was incubated for $20 \mathrm{~min}$ at $37^{\circ} \mathrm{C}$. Sections were then incubated with the biotinylated universal antimouse Ig secondary antibody (Ventana SA, Illkirch, France) for $16 \mathrm{~min}$ at $37^{\circ} \mathrm{C}$, followed by incubation with the avidin-biotinhorseradish peroxidase complex using the Ventana Medical Systems basic DAB detection kit for $8 \mathrm{~min}$ at $37^{\circ} \mathrm{C}$. The final revelation system (DMKiVIEW DAB; Dako) used horseradish peroxidase-diaminobenzidine as the final chromogen.

As negative controls, sections from each specimen were incubated with a normal mouse IgG1 (X0931 culture supernatant; Dako) instead of the primary antibody. Slides were counterstained with haematoxylin.

\section{Microscopy analysis}

We focused our observations on sarcoid granulomas located within the intralobular parenchyma as non-aggregated, isolated granulomas are mostly found in this area. Microscopy analysis comprised of two steps. First, the D2-40-labelled sections were screened and digitalised, and labelled lymphatic vessels in the vicinity of each granuloma were counted. D2-40labelled lymphatics were identified as structures with an open or collapsed lumen, but with a clearly visible endothelial layer. A total of 311 sarcoid granulomas (mean of 54 granulomas per lung section) were studied, 249 of which were located within the pulmonary lobules and 62 along interlobular septa. The morphometric analysis was pursued on a set of 38 digitised images from the microscopy analysis.

Secondly, the distribution of blood microvessels associated with granulomas was analysed using CD34- and CD31-immunolabelled sections. We recorded the presence or absence of associated CD31-labelled blood vessels in each section and counted the number present on a set of 85 strictly intralobular granulomas, which were selected from among those described previously because they were isolated, i.e. non-aggregated early lesions that are easier to examine thoroughly.

\section{Morphometric analysis}

Morphometric analyses were conducted on a set of 38 digitised images of non-coalesced, isolated granulomas adjacent to D240-positive vessels. All images were processed using Nikon NIS-Elements software (Nikon, Apidrag, Bucharest, Romania). The respective areas $(\mathrm{A})$, perimeters $(\mathrm{P})$ and minimum and maximum diameters were then calculated. A script was recorded and automatically run for each image to generate the centre of gravity for each granuloma, referred to as the "centroid". The sphericity index (SI) was calculated as the $\mathrm{d}_{1} / \mathrm{d}_{2}$ ratio $\left(\mathrm{d}_{1}=2 \sqrt{\mathrm{A}} / \pi ; \mathrm{d}_{2}=\mathrm{P} / \pi\right)$, equal to 1 when the structure is a perfect circle, i.e. the cross-section of a sphere. The respective shortest distances from adjacent lymphatics to the proximal border and the "centroid" of the granulomas were then calculated manually.

All data were exported and analysed in Excel (Microsoft Corporation, Redmond, WA, USA).

\section{D-reconstruction of lymphatic-granuloma relationships}

A granuloma located within the lobular parenchyma with a diameter not exceeding $1 \mathrm{~mm}$, so as to be completely visible on all serial sections and easy to follow, was selected for 3Dreconstruction. 12 consecutive D2-40-immunolabelled sections were selected and digitised using Aperio's ScanScope system (Aperio, Vista, CA, USA). Segmentation was obtained by manually drawing the boundaries of the four main components, i.e. clusters of epithelioid cells, the surrounding connective tissue, D2-40-labelled lymphatics and a small satellite artery. The consecutive images were then loaded into WinSurf (SURF driver 4.0; www.surfdriver.com), producing a vectorial 3D-model featuring the coalescent granulomas and their adjacent lymphatics.

\section{RESULTS}

\section{Morphology of sarcoid granulomas}

Typical histopathological features of pulmonary florid sarcoidosis were observed in all six lung biopsies: i.e. compact, noncaseating epithelioid and giant-cell granulomas predominantly located near peribronchovascular bundles, interlobular septa and along the subpleural connective tissue, but also within the lobular parenchyma which was examined in particular detail. Few intralobular granulomas close to or within the alveolar spaces were devoid of a fibrotic ring, while most were surrounded by connective tissue stained blue by Masson's trichrome, suggesting a high type I collagen content (fig. 1).

The distributions of the respective minimum and maximum diameters for a set of 38 intralobular granulomas (fig. 2) demonstrated a minimum diameter exceeding $200 \mu \mathrm{m}$ in up to $37 \%$ of them. The SI of granulomas evaluated after area and perimeter analysis confirmed the constant spheroid-like "rugby ball" or ellipsoid form of them (mean \pm SD $0.86 \pm 0.07$ ).

\section{Lymphatic network-granuloma relationship}

Analysis of the D2-40-immunolabelled sections (table 1) showed that most granulomas $(59 \%$ and $74 \%$ of intralobular and perilobular granulomas, respectively) were adjacent to at least one small lymphatic. The presence of labelled lymphatics in the vicinity of intralobular granulomas is shown in figure 3. Two or more lymphatics were associated with up to $27 \%$ of intralobular granulomas, suggesting their woven basket-like arrangement around granulomas. 

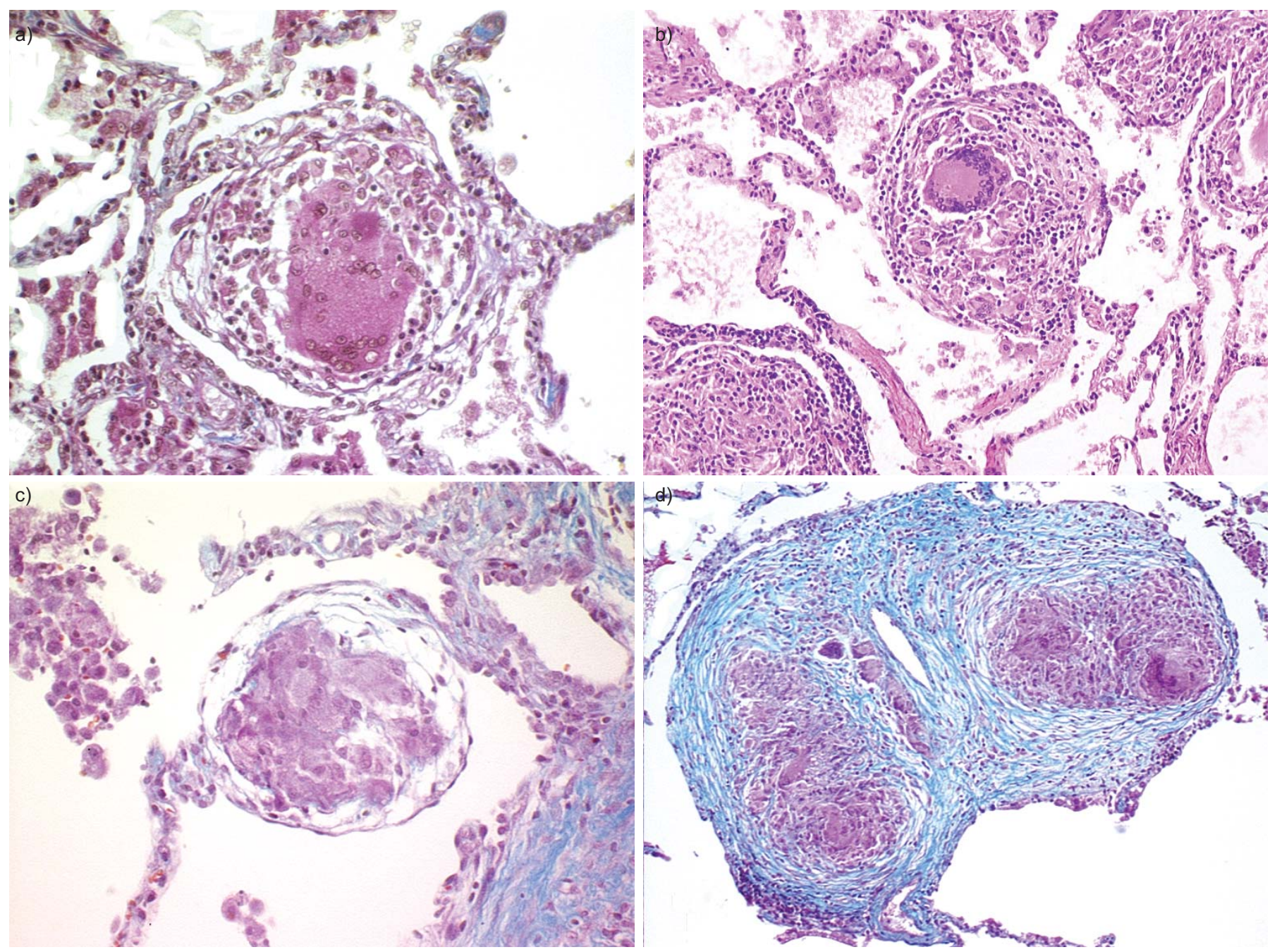

FIGURE 1. a, b) Haematoxylin and eosin or c, d) Masson's trichrome staining of intralobular pulmonary sarcoid granulomas. Note the dense blue stain surrounding two granulomas in (d). Magnifications: a) $\times 350$; b, d) $\times 250$; c) $\times 400$.

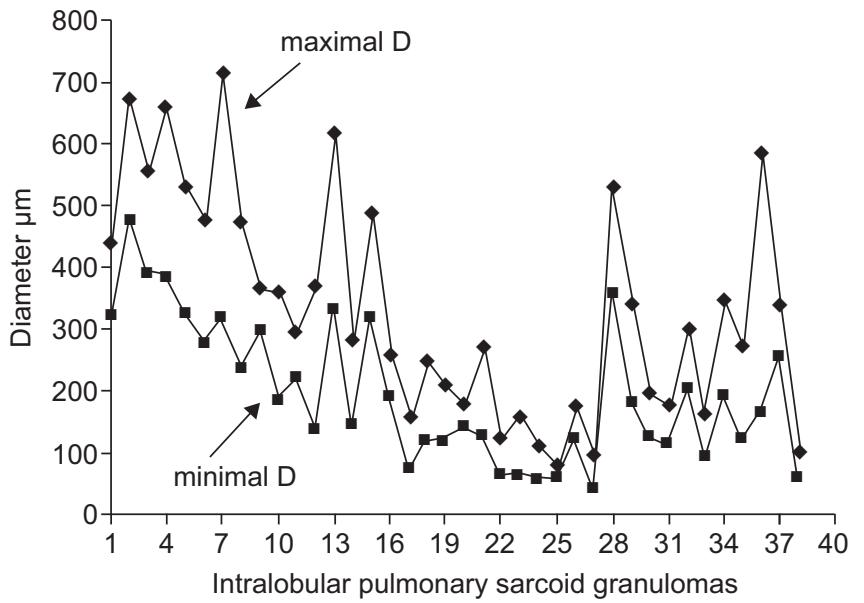

FIGURE 2. Morphometric analysis of the respective minimal (minimal D) and maximal (maximal D) diameters of 38 intralobular pulmonary sarcoid granulomas.

\begin{tabular}{|c|c|c|c|}
\hline TABLE 1 & \multicolumn{3}{|c|}{$\begin{array}{l}\text { Relationship between D2-40-labelled lymphatics } \\
\text { and intralobular or perilobular sarcoid } \\
\text { granulomas. }\end{array}$} \\
\hline \multirow{2}{*}{\multicolumn{2}{|c|}{$\begin{array}{l}\text { Associated D2-40 } \\
\text { lymphatics n }\end{array}$}} & \multicolumn{2}{|c|}{ Granulomas } \\
\hline & & Intralobular\# & Perilobular \\
\hline \multicolumn{2}{|l|}{1} & $80(32)$ & $26(42)$ \\
\hline \multicolumn{2}{|l|}{2} & $37(15)$ & $11(18)$ \\
\hline \multicolumn{2}{|l|}{3} & $17(7)$ & 8 (13) \\
\hline \multicolumn{2}{|l|}{$\geqslant 4$} & $12(5)$ & $1(1)$ \\
\hline \multicolumn{2}{|l|}{ Total } & $147(59)$ & $46(74)$ \\
\hline
\end{tabular}

VOLUME 37 NUMBER 4

837 

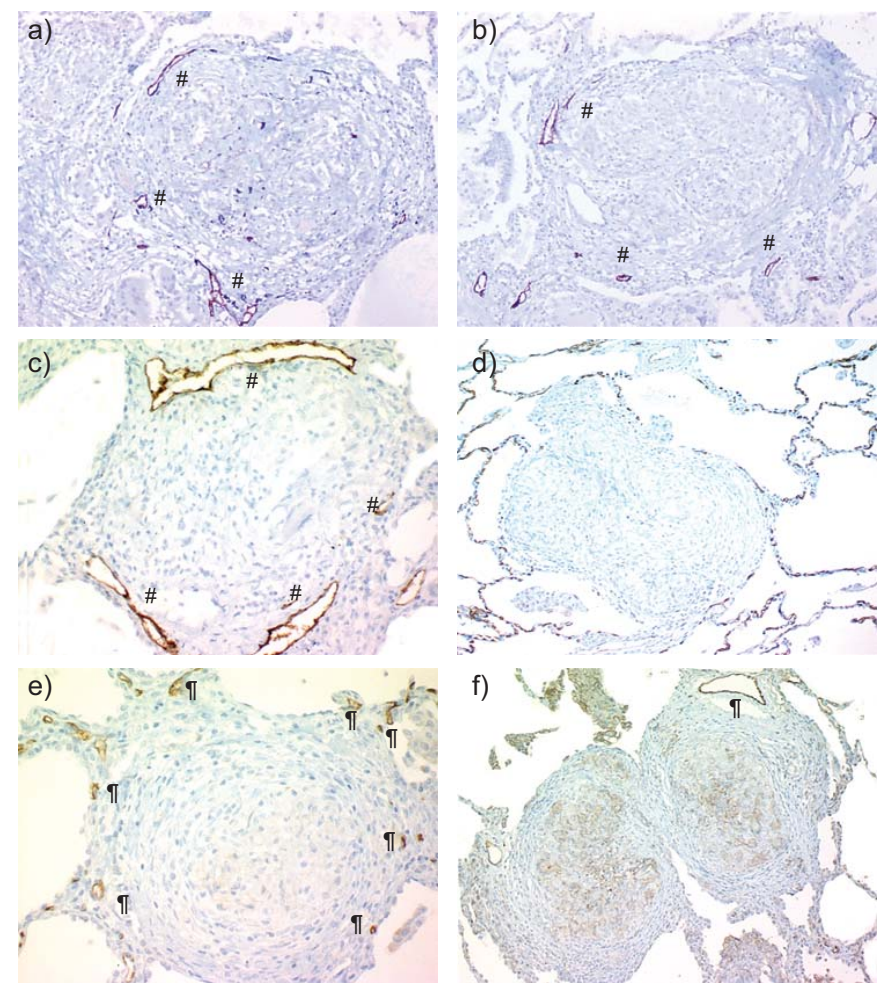

FIGURE 3. a-c) Lymphatic (\#) and d-f) blood microvessels (థ) associated with intralobular sarcoid granulomas. a-c) D2-40 labelled lymphatics near granulomas. d, e) CD34 immunolabelling of blood capillaries. Note the intense CD34 labelling of alveolar capillaries. f) CD31 immunolabelling of a larger blood vessel. Note the absence of small blood capillaries close to the granulomas. Magnification: $a, b, f) \times 350$; c) $\times 500$; d) $\times 300$; e) $\times 400$.

The distance between the closest lymphatic wall and the granuloma border was $<20 \mu \mathrm{m}$ for up to $65 \%$ of intralobular sarcoid granulomas (mean (range) $33 \pm 51 \mu \mathrm{m}(0-256 \mu \mathrm{m}))$. The distance between the "granuloma centroid" and the next lymphatic wall exceeded $150 \mu \mathrm{m}$ for $63 \%$ of the lesions $(182 \pm 97 \mu \mathrm{m}(23-431 \mu \mathrm{m}))$. No lymphatic vessel was seen within granulomas.

\section{D-reconstruction of a sarcoid granuloma and its surrounding lymphatic network}

The 3D-reconstruction model (fig. 4) clearly shows the internal structure of a sarcoid granuloma which consists of small clusters of closely packed epithelioid and giant cells surrounded by a connective tissue sheath traversed by the lymphatic network. No lymphatic network was observed within the cellular part of the granuloma.

\section{Blood microvascular network-granuloma relationship}

Other than a few juxta-alveolar granulomas, blood capillaries were rarely seen in the vicinity of granulomas (fig. 3 ). The CD34- or CD31-labelled blood-microvessel network of the interalveolar walls was stopped in contact with the outer fibrous ring of sarcoid granulomas. Because CD31 labelling of juxta-granuloma blood vessels was more intense than CD34 labelling, quantitative analysis was performed on CD31immunolabelled sections, analysing 85 granulomas and their environment. CD31-labelled blood microvessels were only

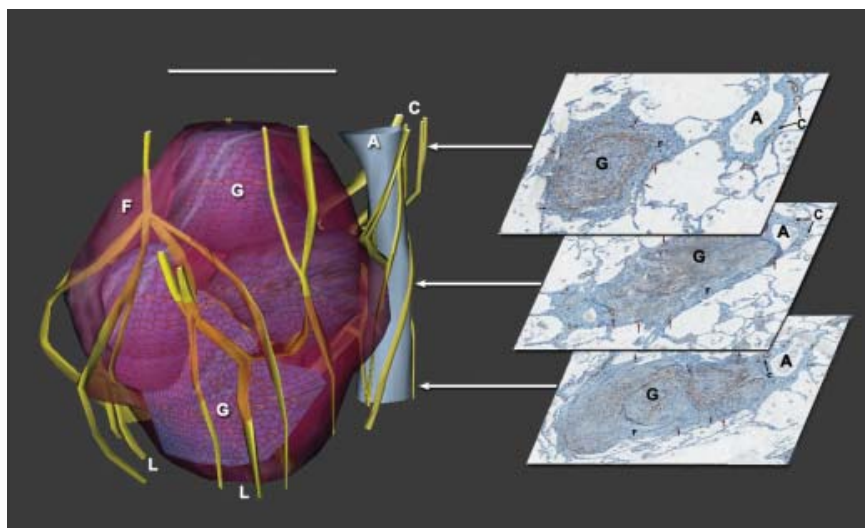

FIGURE 4. Three-dimensional (3D) reconstruction of granulomas located within a pulmonary lobule. 12 D2-40-immunolabelled serial sections were used for 3D modelling of the lesion. The clusters of the cellular components of granulomas (G) are pink, whereas the surrounding connective tissue sheath $(F)$ is purple. D2-40 labelled lymphatic channels $(\mathrm{L})$ and lymphatic collectors $(C)$ are yellow and the satellite intralobular artery $(A)$ is blue. Scale bar $=500 \mu \mathrm{m}$.

observed in $15 \%$ of the granulomas, at the periphery of the perigranuloma fibrous ring. A larger CD31-positive blood vessel, identified as an intralobular vein or artery, weakly immunolabelled for CD34, was seen close to the perigranuloma fibrous ring of $25 \%$ of intralobular granulomas; therefore, at least $75 \%$ of granulomas were devoid of blood vessels in their immediate vicinity.

No blood capillary or larger vessel was seen in either the central part of granulomas or their outer fibrous ring.

\section{DISCUSSION}

The lymphatic distribution of pulmonary sarcoid granulomas is a well-known and constant histopathological feature of sarcoidosis and, thus, serves as a valuable diagnostic criterion for it [2, 6]. However, until now, the intimate morphological relationships between pulmonary sarcoid granulomas and the lymphatic network had never been analysed in detail. Although the tropism of pulmonary sarcoid granulomas for the blood vessel walls has been widely described, little is known about the pattern of the blood microvessel supply in the immediate vicinity of sarcoid granulomas [3]. We thought that better comprehension of the relationships between individual sarcoid lesions and their lymphatic or blood microvascular networks might provide insights into the pathophysiology of the disease and help design future therapeutics.

Using samples of pulmonary surgical biopsies, we first demonstrated that most of the intra- and perilobular sarcoid granulomas were associated with at least one lymphatic. Notably, the scattered intralobular granulomas were connected to the lymphatic network, supporting the hypothesis of the critical role of lymphatics in the pathophysiology of the disease. In addition, CD31 and CD34 immunolabelling of the same samples showed that the granulomas were very poorly supplied by blood capillaries at the outer edge of the peripheral fibrous ring, far from the cellular compartment.

In most open lung biopsies obtained in the setting of pulmonary sarcoidosis, granulomas surrounded by fibrotic 
rings coalesced to form a nodule, thereby perturbing the topographic lobular distribution of lesions. Therefore, in order to obtain a meticulous evaluation of the topographic markings of each granuloma we intentionally focused our study on solitary lesions usually observed within the intralobular parenchyma, away from the sarcoid nodules. It is important to keep in mind that the technological advances of immunohistochemistry only recently enabled the thin lymphatic network supplying the collecting lymphatics to be described in detail [4].

Pulmonary lymphatics play a pivotal role in regulating the drainage of alveolar fluids throughout the interstitial framework under physiological conditions and following lung injury [7-9]. Importantly, a wide range of small particles including organic and inorganic dusts, and infectious microorganisms are processed and drained by the lymphatics reaching the collecting channels located within perivenular and peribroncho-arterial sheaths [7-9]. Indeed, some studies particularly emphasised the major role of the lymphatics in granuloma formation after alveolar processing of airborne pathogens. In guinea pigs experimentally infected by aerosols containing Mycobacterium tuberculosis, it was demonstrated that the thin connective tissue space between the alveolar membrane and the lymphatic was the selective area for inflammatory cell clustering, giving rise to epithelioid and giant-cell granulomas [10]. The role of airborne environmental factors in the pathogenesis of pulmonary sarcoidosis is strongly suspected [11-15]. A prospective study conducted on patients diagnosed with pulmonary sarcoidosis revealed a link with beryllium exposure in a significant percentage of them [16]. Moreover, a relationship between sarcoidosis and exposure to the crystalline silica cristobalite was demonstrated in the population of an Icelandic district [11]. Similarly, sarcoid-like pulmonary granulomatosis has been reported in New York firefighters, following the World Trade Center Disaster [12] More recently, specific attention was paid to the role of pulmonary lymphatics in the clearance of inhaled ultrafine and nanosized particles $<100 \mathrm{~nm}$ [17]. In contrast with larger particles, once deposited, these nanoparticles readily translocate to extrapulmonary sites [17]. Indeed, it could be tempting to suggest the role of such particles as a triggering factor in the granulomatous process of sarcoidosis, a systemic disease with major pulmonary and node involvement.

We did not observe any lymphatics within granulomas. Although the walls of lymphatics constantly adhered to the thin fibrotic ring enveloping granulomas, lymphatic lumens seem to have merely been displaced from their native course by sarcoid lesions. Morphometric data on SI and the distances from the granuloma "centroid" to the closest lymphatic vessels support this type of granulomatous concentric growth in contact with, but not integrating lymphatics. Similarly, a previous ultrastructural study performed on serial ultra-thin sections containing a pulmonary sarcoid granuloma identified a lymphatic within the peripheral connective tissue, but none within the granulomatous cell component [18]. Those authors described the presence of lymphocytes and mononuclear cells within this lymphatic running along the outside of the perigranuloma fibrous ring, but the direction of its flow could not be determined [18]. Granulomas are composed of central clusters of epithelioid cells and giant cells primarily associated with peripheral T lymphocytes [19]. All these observations strongly support a major role of juxta-granuloma lymphatics in lymphocyte-trafficking towards mediastinal lymph nodes. The mobilisation of $\mathrm{CD}^{+} \mathrm{T}$ lymphocytes, known to play a pivotal role in the granulomatous process of sarcoidosis via the lymphatics, might explain the onset of granulomas along the lymphatic collectors flowing towards the mediastinal lymph nodes [19].

Except for a very few small-diameter granulomas localised in the interalveolar walls, capillary CD31 and CD34 immunolabelling showed blood microvascularisation confined at the outer border of the connective tissue ring, far from the cellular compartment in up to $85 \%$ of sarcoid granulomas. This finding suggests that a large majority of pulmonary sarcoid granulomas can be considered "avascular". These observations, along with previous findings, give rise to three hypotheses. First, we did not observe any angiogenesis in pulmonary sarcoidosis, in contrast with other pulmonary inflammatory fibrotic processes [20]. This absence of angiogenesis is at variance with the expected findings, in light of the high levels of vascular endothelial growth factor in bronchoalveolar lavage fluids reported in sarcoidosis, and in epithelioid cells detected by immunohistochemistry and in situ hybridisation [21, 22]. Conversely, macrophages are also known to secrete anti-angiogenic factors, such as endostatin/ collagen XVIII [23]. However, the secretion of such factors by epithelioid or giant cells has not been reported.

Secondly, more than half of the granulomas we examined exceeded $200 \mu \mathrm{m}$ in diameter, leading us to think that the distance separating them from the blood capillaries could be responsible for intragranuloma hypoxia. Indeed, it was shown that the oxygen tension $\left(\mathrm{PO}_{2}\right)$ gradient in tissues dropped exponentially as a function of the distance from the blood vessels [24]. In the present context, such a hypothetical relationship between the granuloma $\mathrm{PO}_{2}$ gradient and the distance from blood vessels and/or alveolar spaces could be advanced. The evidence suggests that the centre of aggregated granulomas, impacted in a thick fibrous sheath, is much further from the blood vessels and, therefore, even more hypoxic. The concept of "hypoxic granuloma" in sarcoidosis is supported by previously described hypoxaemic granulomas in the setting of experimental tuberculosis in guinea pigs [25]. Such a hypothesis could also explain, at least in part, perigranuloma fibrosis, the hallmark of sarcoid granuloma progression [2, 6, 26-29]. In this hypoxic context, transforming growth factor- $\beta$, one of the cytokines secreted by sarcoid granulomas, has been shown to exert a synergistic action on collagen production by fibroblasts [28, 29]. Moreover, it is striking that a minority of small diameter interalveolar granulomas close to blood capillaries or alveolar spaces were not associated with a fibrous ring.

Thirdly, the distance between capillaries and the cellular component of granulomas might hamper the distribution of systematically administered drugs used to treat sarcoidosis. Indeed, a collagen-rich area including a well-defined collagen network organised like a "capsule" was demonstrated to facilitate the interstitial transport of fluid and molecules [30].

In conclusion, this focused examination of the morphological features of the local lymphatic environment of intralobular pulmonary sarcoid granulomas provided new evidence for the 
critical role of lymphatics in the emergence of these lesions, and reinforced the putative role of airborne particles in their pathogenesis. Furthermore, the consequences of the scarce blood microvessels on the role of hypoxia in granulomas or impairment of therapeutic drug distribution can be envisaged.

\section{STATEMENT OF INTEREST}

A statement of interest for D. Valeyre can be found at www.erj. ersjournals.com/site/misc/statements.xhtml

\section{ACKNOWLEDGEMENTS}

We are grateful to L. Germain (Histology Dept, ER2 Université Pierre et Marie Curie, Paris, France) and B. Lejeune (Pathology Dept, Hôpital Avicenne, AP-HP, Bobigny, France) for their technical help and to A. Martin (Pathology Dept, Hôpital Avicenne, AP-HP), P. Callard (Pathology Dept, Hôpital Tenon ER2 Université Pierre et Marie Curie) and the Amis du Centre des Tumeurs de Tenon (Pavillon Proust Hôpital Tenon, Paris) for their constant support. We also thank J. Jacobson (translator; Paris, France) for editorial assistance.

\section{REFERENCES}

1 Iannuzzi MC, Rybicki BA, Teirstein AS. Sarcoidosis. N Engl J Med 2007; 357: 2153-2156.

2 Idiopathic interstitial pneumonia and other diffuse parenchymal lung diseases. In: Travis MD, Colby TV, Koss MN, et al, eds. Atlas of Non-Tumor Pathology 2. Non-Neoplastic Disorders of the Lower Respiratory Tract. 2nd Edn. Washington DC, Armed Forces Institute of Pathology, 2002; pp.123-136.

3 Takemura T, Matsui Y, Saiki S, et al. Pulmonary vascular involvement in sarcoidosis. Hum Pathol 1992; 23: 1216-1223.

4 Kambouchner M, Bernaudin JF. Intralobular pulmonary lymphatic distribution in normal human lung using D2-40 antipodoplanin immunostaining. J Histochem Cytochem 2009; 57: 643-648.

5 Pusztaszeri MP, Seelentag W, Bosman FT. Immunohistochemical expression of endothelial markers CD31, CD34, von Willebrand factor, and Fli-1 in normal human tissues. J Histochem Cytochem 2006; 54: 385-395.

6 Ma YL, Gal A, Koss MN. The pathology of pulmonary sarcoidosis: update. Semin Diagn Pathol 2007; 24: 150-161.

7 Leak LV. Lymphatic removal of fluids and particles in the mammalian lung. Environ Health Perspect 1980; 35: 55-75.

8 Lauweryns JM, Baert JH. Alveolar clearance and the role of the pulmonary lymphatics. Am Rev Respir Dis 1977; 115: 625-683.

9 Macklin C. Lung fluid, alveolar dust drift and initial lesions of disease in the lung. CMAJ 1955; 72: 664-665.

10 Basaraba RJ, Smith EE, Shanley CA, et al. Pulmonary lymphatics are primary sites of Mycobacterium tuberculosis infection in guinea pigs infected by aerosol. Infect Immun 2006; 74: 5397-5401.

11 Rafnsson $\mathrm{V}$, Ingmarsson $\mathrm{O}$, Hjalmarsson $\mathrm{I}$, et al. Association between exposure to crystalline silica and risk of sarcoidosis. Occup Environ Med 1998; 55: 657-660.

12 Izbicki G, Chavko R, Banauch GI, et al. World Trade Center "sarcoid-like" granulomatous pulmonary disease in New York City Fire Department rescue workers. Chest 2007; 131: 1414-1423.
13 Statement on sarcoidosis. Joint Statement of the American Thoracic Society (ATS), the European Respiratory Society (ERS) and the World Association of Sarcoidosis and Other Granulomatous Disorders (WASOG) adopted by the ATS Board of Directors and by the ERS Executive Committee, February 1999. Am J Respir Crit Care Med 1999; 160: 736-755.

14 Martin WJ, Iannuzzi MC, Gail DB, et al. Future directions in sarcoidosis research. Summary of an NHLBI working group. Am J Respir Crit Care Med 2004; 170: 567-571.

15 Jajosky P. Sarcoidosis diagnoses among US military personnel: trends and ship assignment associations. Am J Prev Med 1998; 14: 176-183.

16 Müller-Quernheim J, Gaede KI, Fireman E, et al. Diagnosis of chronic beryllium disease within cohorts of sarcoidosis patients. Eur Respir J 2006; 27: 1190-1195.

17 Oberdörster G, Oberdörster E, Oberdörster J. Nanotoxicology: an emerging discipline evolving from studies of ultrafine particles. Environ Health Perspect 2005; 113: 823-839.

18 Soler P, Basset F, Bernaudin JF, et al. Morphology and distribution of the cells of a sarcoid granuloma: ultrastructural study of serial sections. Ann NY Acad Sci 1976; 278: 147-160.

19 Grunewald J, Eklund A. Role of $\mathrm{CD}^{+}{ }^{\mathrm{T}}$ cells in sarcoidosis. Proc Am Thorac Soc 2007; 4: 461-464.

20 Tzouvelekis A, Anevlavis S, Bouros D. Angiogenesis in interstitial lung diseases: a pathogenetic hallmark or a bystander? Respir Res 2006; 7: 82.

21 Vasakova M, Sterclova M, Kolesar L, et al. Bronchoalveolar lavage fluid: cellular characteristics, functional parameters and cytokine and chemokine levels in interstitial lung diseases. Scand J Immunol 2009; 69: 268-274.

22 Tolnay E, Kuhnen C, Voss B, et al. Expression and localization of vascular endothelial growth factor and its receptor FLT in pulmonary sarcoidosis. Virchows Arch 1998; 432: 61-65.

23 Paddenberg R, Faulhammer P, Goldenberg A, et al. Hypoxiainduced increase of endostatin in murine aorta and lung. Histochem Cell Biol 2006; 251: 497-508.

24 Torres Filho IV, Leuning M, Yuan F, et al. Non invasive measurement of microvascular and interstitial oxygen profiles in a human tumor in SCID mice. Proc Natl Acad Sci USA 1994; 91: 2081-2085.

25 Via LE, Lin PL, Ray SM, et al. Tuberculous granulomas are hypoxic in guinea pigs, rabbits and non-human primates. Infect Immun 2008; 76: 2333-2340.

26 Higgins DF, Kimura K, Iwano M, et al. Hypoxia-inducible factor signalling in the development of tissue fibrosis. Cell Cycle 2008; 7: $1128-1132$.

27 Tzouvelekis A, Harokopos V, Paparountas T, et al. Comparative expression profiling in pulmonary fibrosis suggests a role of hypoxia-inducible factor-1alpha in disease pathogenesis. Am J Respir Crit Care Med 2007; 176: 1108-1119.

28 Falanga V, Zhou L, Yufit T. Low oxygen tension stimulates collagen synthesis and COL1A1 transcription through the action of TGF- 31 . J Cell Physiol 2002; 191: 42-50.

29 Salez F, Gosset $\mathrm{P}$, Copin MC, et al. Transforming growth factor- $\beta 1$ in sarcoidosis. Eur Respir J 1998; 12: 913-919.

30 Netti PA, Berk DA, Swartz MA, et al. Role of extracellular matrix assembly in interstitial transport in solid tumors. Cancer Res 2000; 60: 2497-2503. 